\title{
Manejo seguro de opioides usados no cuidado paliativo: revisão narrativa
}

\author{
Safe management of opioids used in palliative care: a narrative review
}

Manejo seguro de opioides usados en cuidados paliativos: una revisión narrativa

Letícia de Paula Santos $^{1 *}$, Lívia Hermsdorff Martins Fontoura ${ }^{1}$, Samuel Felipe Almeida Silva ${ }^{1}$, Ana Luiza Brandão Santos ${ }^{2}$, Georgia Luiza Caires Tanajura ${ }^{1}$, Gabriel Calafange Cunha ${ }^{3}$, Ana Luiza Faria Rabelo ${ }^{3}$, Camilla de Freitas Maziero ${ }^{3}$, Larissa Mariani Rezende Almeida ${ }^{3}$, Jacqueline Souza Dutra Arruda¹.

\section{RESUMO}

Objetivo: Apresentar informações sobre o mecanismo de ação, indicações e contraindicações dos principais opioides usados no cuidado paliativo, subsidiando a prescrição correta e segura. Revisão bibliográfica: Os opioides são drogas de ação analgésica eficaz para dores moderadas ou graves, apesar da sua eficácia, existe resistência em seu uso tanto por parte dos médicos quanto dos pacientes, já que pode causar dependência física, depressão respiratória, dentre outros efeitos colaterais indesejados. Dessa forma, sintetizar sobre estes medicamentos permite que haja uma avaliação crítica para que o melhor plano terapêutico seja ofertado ao paciente. Comumente, a morfina é o opioide mais utilizado, o fentanil é o mais potente e a metadona possui o maior tempo de ação. A indicação de cada um deve ser bem avaliada de acordo com o plano terapêutico proposto pela equipe multidisciplinar, isso porque o controle da dor não é alcançado apenas com o uso de analgésico, já que possui componentes bio-psicossociais e espirituais que devem ser trabalhados. Considerações finais: Determinou-se a importância de conhecer o mecanismo de ação dos opioides, com finalidade de intervir precocemente nos efeitos adversos e conferir maior segurança na sua administração, obtendo, assim, efeito direto sobre a qualidade de vida durante o cuidado paliativo.

Palavras-chave: Opioides, Dor, Cuidado paliativo.

\begin{abstract}
Objective: To present information on the mechanism of action, indications and contraindications of the main opioids used in palliative care, supporting the correct and safe prescription. Bibliographic review: Opioids are effective analgesic drugs for moderate or severe pain, despite their effectiveness, there is resistance to their use by both physicians and patients, as it can cause physical dependence, respiratory depression, among other unwanted side effects. Thus, synthesizing about these drugs allows for a critical assessment so that the best therapeutic plan is offered to the patient. Morphine is commonly the most used opioid, fentanyl is the most potent and methadone has the longest action time. The indication of each should be well evaluated according to the therapeutic plan proposed by the multidisciplinary team, because pain control is not achieved only with the use of analgesics, as it has bio-psychosocial and spiritual components that must be worked on. Final considerations: The importance of knowing the mechanism of action of opioids was determined, in order to intervene early in adverse effects and provide greater safety in their administration, thus obtaining a direct effect on the quality of life during palliative care.
\end{abstract}

Keywords: Opioids, Pain, Palliative care.

\footnotetext{
${ }^{1}$ Centro Universitário de Caratinga (UNEC), Caratinga - MG. *E-mail: leledepaulasantos@gmail.com

2 Faculdade Única de Ipatinga (ÚNICA), Ipatinga - MG.

${ }^{3}$ Faculdade de Medicina do Vale do Aço (UNIVAÇO), Ipatinga - MG.
} 


\section{RESUMEN}

Objetivo: Presentar información sobre el mecanismo de acción, indicaciones y contraindicaciones de los principales opioides utilizados en cuidados paliativos, sustentando la prescripción correcta y segura. Revisión bibliográfica: Los opioides son fármacos analgésicos eficaces para el dolor moderado o severo, a pesar de su eficacia, existe resistencia a su uso tanto por parte de médicos como de pacientes, ya que puede provocar dependencia física, depresión respiratoria, entre otros efectos secundarios no deseados. Así, sintetizar sobre estos fármacos permite una evaluación crítica para ofrecer al paciente el mejor plan terapéutico. La morfina es comúnmente el opioide más utilizado, el fentanilo es el más potente y la metadona tiene el tiempo de acción más prolongado. La indicación de cada uno debe ser bien valorada según el plan terapéutico propuesto por el equipo multidisciplinario, pues el control del dolor no se logra solo con el uso de analgésicos, ya que tiene componentes biopsicosociales y espirituales sobre los que hay que trabajar. Consideraciones finales: Se determinó la importancia de conocer el mecanismo de acción de los opioides, con el fin de intervenir precozmente en los efectos adversos y brindar mayor seguridad en su administración, obteniendo así un efecto directo sobre la calidad de vida durante los cuidados paliativos.

Palabras clave: Opioides, Dolor, Cuidados paliativos.

\section{INTRODUÇÃO}

O cuidado paliativo é aquele que proporciona alívio dos desconfortos gerados por doenças em estágios avançados. O amplo conhecimento sobre esses cuidados faz-se necessário a todos os profissionais de saúde, já que, o tratamento humanizado proporciona ao paciente uma melhor sobrevida e conforto (COELHO CB e YANKASKAS JR, 2016).

A dor possui efeito direto sobre a qualidade de vida, portanto, a assistência intervencionista e curativa, com a utilização apenas de analgésicos, por exemplo, não é suficiente para o seu controle, exigindo um cuidado holístico, voltado para o componente bio-psicossocial-espiritual tanto para o paciente acometido quanto para seus familiares (ARAÚJO LG, et al., 2020). Para essa complexa abordagem, são necessários uma equipe multidisciplinar e a utilização de instrumentos padronizados para compreender o sofrimento do paciente e auxiliar na elaboração de um plano terapêutico, como a escala visual analógica (EVA) e a escala de dor Leeds Assessment of Neuropathic Symptoms and Signs (LANSS) (WIERMANN EG, et al., 2014).

Para avaliar a intensidade da dor do paciente, o melhor parâmetro de avaliação é a EVA, esta considera a expressão facial do paciente e a dor autodeclarada em uma escala numérica de 0 a 10, onde 0 representa a ausência de dor, e 10 representa a pior dor imaginável. Já para a avaliação do tipo da dor, a LANSS é uma ferramenta de alta eficácia para distinguir dor nociceptiva, neuropática e mista, ela vai de 0 a 24 e possui duas seções, uma qualitativa e outra com os aspectos sensitivos da dor (BRASIL, 2012). Dessa forma, a equipe médica consegue monitorar cuidadosamente a dor do paciente, possibilitando a tomada de decisão para amenizar os desfechos negativos e proporcionar mais conforto (SANTOS AD, et al., 2020).

Uma das formas de controle da dor é a utilização de opioides, que apesar de altamente eficaz deve ser cuidadosamente indicada, para isso deve haver a participação do serviço clínico farmacêutico, bem como de toda a equipe multiprofissional. Os opioides auxiliam no controle da dor moderada e grave, que dentro das escalas numéricas da dor correspondem a pontuações iguais ou maiores que quatro pontos, para dores leves, ou seja, aquelas com pontuações menores que quatro, outros analgésicos ou medidas não farmacológicas podem ser utilizadas, sendo que todos precisam ser devidamente acompanhados pela equipe multidisciplinar. Vale ressaltar que o tratamento insuficiente da dor, causam prejuízos em relação ao sono e as funções cognitivas, resultando em ansiedade, depressão, e afetando a qualidade de vida do paciente como um todo, tendo um grande impacto negativo no conforto do enfermo (AZEVEDO AS, 2017; LEÓN MX, et al., 2019).

Apesar da evidente importância dos opioides, os seus efeitos adversos são responsáveis pela resistência a sua prescrição, podemos citar a Hiperalgesia Induzida por Opioides (HIO), que ocorre principalmente na dor crônica não maligna; a depressão respiratória, a euforia e a tolerância. A tolerância pode resultar em 
dependência física, ocasionando quadros de abstinência, que possuem como principais sintomas: insônia, cãibras, diarreia, náusea, vômito, dores no corpo, disforia, ansiedade e irritabilidade (PETER YI e PRYZBYLKOWSKI P, 2015; LEAL RS e ALENCAR GA, 2020). Além disso, os opioides podem causar sintomas gastrointestinais, como constipação, anorexia e refluxo gastroesofágico (ORIOL AL, et al., 2018).

Uma pesquisa de Calônego MA (2020) indica a existência de vários motivos que dificultam a prescrição de opioides, como por exemplo, o receio de causar uma iatrogenia médica, por medo de induzir o paciente ao vício $(52,19 \%)$, a dificuldade de obtenção do receituário "Tipo $A$ " $(50,13 \%)$, a falta de conhecimento farmacológico (42,67\%), além do medo apresentado pelos pacientes do vício (86,59\%) e a falta de preparo médico para lidar com a dor dos pacientes paliativos $(58,61 \%)$, ou seja, é evidente que existem dificuldades sociais, legais e burocráticas para a prescrição dos medicamentos derivados do ópio. Torna-se evidente, portanto, a necessidade de mais pesquisas voltadas ao tratamento da dor no cuidado paliativo.

O objetivo deste presente estudo é apresentar informações sobre o mecanismo de ação, indicações e contraindicações dos principais opioides usados no cuidado paliativo, subsidiando a prescrição correta e segura.

\section{REVISÃO BIBLIOGRÁFICA}

Para fazer o correto manejo dos opioides, é imprescindível o conhecimento de seu mecanismo de ação, que consiste na ativação de receptores localizados no sistema nervoso central (SNC), sendo que existem três tipos de receptores onde os opioides se ligam: Mu, kappa e delta. Estes receptores estão acoplados a proteínas $\mathrm{G}$ inibitórias (Gi), que inibem a adenilato ciclase, e consequentemente, diminui a quantidade de adenosina 3',5'-monofosfato cíclico (AMPc), afetando a função celular. Dessa forma, os opioides promovem a abertura de canais de potássio e a célula fica hiperpolarizada. Simplificando, os opioides atuam hiperpolarizando os neurônios, diminuindo sinapses e reduzindo a síntese proteica, alterando a função celular do neurônio, que paralisa a passagem de informações referente à dor (RANG, et al., 2016).

Além disso, atuam em várias funções fisiológicas, como exemplo na modulação do trato gastrointestinal, no sistema endócrino, na aprendizagem e na memória. Atuam ainda no sistema de recompensa do SNC causando euforia, pois liberam dopamina no núcleo accumbens, o que inibe a secreção de ácido gamaaminobutílico (GABA) pelos neurônios da área tegmentar ventral (GOODMAN A, et al., 2018).

A codeína é um opioide fraco, que é obtido naturalmente pela extração da planta Papaver somniferum. Essa droga possui menor tendência em causar tolerância em relação aos principais opioides, além disso, é considerada um pró-fármaco clássico, pois aumenta a biodisponibilidade e diminui a toxicidade. Geralmente, é administrada por via oral e é metabolizada em morfina pelo fígado (GOODMAN A, et al., 2018).

Portanto, podemos concluir que pacientes com distúrbios da deglutição, doenças hepáticas e diarreia persistente, não são indicados a receberem está medicação. Comumente, faz-se a prescrição de $30 \mathrm{mg}$ de Codeína em associação a $500 \mathrm{mg}$ de Paracetamol para dores moderadas, principalmente após traumas e em pós-operatório; entretanto, deve-se manejar essas drogas cuidadosamente, pois não é recomendado a pacientes com insuficiência hepática, gestantes, lactantes e não há eficácia para crianças menores de 12 anos (WALTER IT, 2019)

A hidrocodona (ou dihidrocodona) é uma droga sintetizada a partir da codeína, sendo muito utilizada nos Estados Unidos da América (EUA) para o manejo da dor crônica, pois proporciona alívio da dor de forma eficaz em pacientes idosos, no entanto, essa droga causa náuseas e constipação (OLIVÊNCIA AS, et al., 2018).

O tramadol é um opioide fraco com um mecanismo de ação exclusivo, pois ele possui pouca afinidade pelos receptores $\mathrm{Mu}$, e seu mecanismo consiste em inibir a recaptação de noradrenalina e serotonina na via descendente da dor. Ele é administrado por via oral, via intravenosa ou supositório retal, sendo indicado para dores moderadas e a dosagem diária recomendada é de 25 a $100 \mathrm{mg}$ a cada 4 a 6 horas (GONG L, et al., 2014). Além disso, sabe-se que os principais efeitos colaterais desta medicação são: boca seca, náusea e tontura. Ressalta-se, ainda, que, para os pacientes oncológicos adultos, o tramadol é eficaz para o alívio da 
dor, mas não há evidências que confirmem a sua eficácia em crianças. Ademais, ele é vantajoso por ter baixo risco de abuso (mas não é nulo) e por ter o seu regulamento de controle menos rigoroso quando comparado a opioides mais potentes (WIFFEN PJ, et al., 2017).

O tapentadol possui estrutura similar ao tramadol, por isso, ele atua como opioide, mas também bloqueia a recaptação de noradrenalina e serotonina. Essa droga é eficaz no manejo de dor nociceptiva ou neuropática descritas pelo paciente como moderada a grave. Todavia, possui efeitos colaterais como: náuseas, vômitos e tonturas (CARDOSO Al, 2013). No entanto, o índice de prescrições deste fármaco está crescendo juntamente com o seu mau uso. Assim sendo, os potenciais efeitos toxicológicos do tapentadol ainda estão em estudo. Novos estudos sugerem que ele possa causar efeitos colaterais neurológicos, cardíacos e pulmonares, quando prescrita a dose máxima diária por um período maior que 14 dias (BARBOSA J, et al., 2021).

A meperidina é um opioide sintético, que possui efeito analgésico, ação espasmolítica e anti-histamínica suave. É administrada por via intramuscular, mas também pode ser administrada por via oral. Ela é metabolizada pelo fígado e o seu principal metabólito é a normeperidina, que é excretada pelo trato urinário. De forma geral, ela possui curta duração, não causa miose, mas pode causar dependência precoce, sendo mais utilizada em dores agudas (BOTAN AG e LAPENA SA, 2015).

Naturalmente, a taxa de metabolização hepática e a taxa de filtração renal diminuem com a idade, consequentemente, deve-se manejar as drogas metabolizadas pelo fígado e excretadas pelos rins cuidadosamente em pacientes idosos. A diminuição da atividade do citocromo P450 no fígado pode resultar em acumulo de opioides na corrente sanguínea e levar a um quadro de intoxicação, por isso, é necessário monitorar os pacientes com idade acima de 60 anos, para fazer os ajustes de dose quando for preciso (NAPLES JG, et al., 2016).

A morfina é um opioide muito usado na dor aguda e crônica, sendo que em 2005 a Organização Mundial da Saúde (OMS) estabeleceu regras para o manejo da mofina para o alivio da dor. Ela possui rápida absorção por via oral, é metabolizada pelo fígado e excretada de forma ativa pelos rins, sendo muito utilizada em pacientes com dor moderada a grave, pois possui rápida liberação com início da sua ação em 30 minutos e duração de 4 horas (SANTOS MH, 2019).

Evidências indicam que cerca de 30 a $40 \%$ dos pacientes em tratamento oncológico curativo e em 70 a $90 \%$ dos pacientes em fase avançada do câncer sentem a presença da dor, sendo que apenas 10 a $30 \%$ destes pacientes recebem o tratamento adequado para o alívio desta dor, o que ressalta a importância de saber prescrever a morfina para a melhora da qualidade de vida destes pacientes. Em adultos, a dose inicial é de $5 \mathrm{mg}$ a cada 4 horas por via oral e a potência relativa da morfina por via intravenosa em relação à por via oral é de 1:3, em relação à subcutânea é de 1:2 (RIVEROS ME e AYALA SJ, 2020).

A hidromorfina também é um opioide que pode ser utilizado nos casos de pacientes com dor moderada a intensa, porém, estudos demonstram que sua potência analgésica é semelhante à da morfina e da oxicodona e, além disso, os efeitos colaterais também são semelhantes. A prescrição de hidromorfina é vantajosa para alivio da dor durante o sono, para aqueles pacientes oncológicos que têm dificuldade em dormir em decorrência da dor (BAO YJ, et al., 2016). Além disso, ela é prescrita nos casos de dor aguda, em administração peridural, pois chega rapidamente ao corno dorsal da medula e tem efeito analgésico rapidamente, com duração de até 6 a 14 horas, sem precisar de dose de resgate. Entretanto, essa técnica é feita por especialistas em ambiente hospitalar (OSORIO-GUTIÉRREZ Cl, et al., 2021).

A metadona é um opioide lipossolúvel de meia vida longa, utilizada principalmente em dor oncológica. É administrada por via oral, sendo absorvida completamente e rapidamente por esta via. Por ser uma substancia lipofílica, é capaz de atravessar a barreira hematoencefálica. Em relação à morfina, ela possui maior biodisponibilidade, maior meia vida, maior ligação plasmática e não produz metabolitos ativos (ou seja, sofre menos influência por disfunção renal); todavia, a metadona sofre mais influência pela disfunção hepática (NETO JO, et al., 2015). Ela é um opioide sintético, e seu efeito analgésico dura de 6-8 horas, mas pode chegar a 24 horas, sendo que a metadona é 10 vezes mais potente que a morfina, além disso, provoca menos náuseas, constipação e sedação (SILVA LJ, et al., 2020). 
A oxicodona teve seu consumo aumentado nas últimas décadas, principalmente nos EUA e na Filândia. Este fármaco pode ser administrado de forma oral, intramuscular ou intravenosa, sendo que a molécula se liga a albumina para ser distribuída e, posteriormente, é excretada de forma ativa pelos rins, também atravessa a barreira placentária e é excretada no leite materno. Além disso, idosos em uso de oxicodona apresentam frequentemente episódios de apneia. Dessa forma, é um medicamento que deve ser prescrito e administrado cautelosamente (PIIRAINEN P, et al., 2019). Para adultos com dor oncológica, a oxicodona não oferece vantagens analgésicas quando comparada a morfina, sendo assim, ambas as drogas podem ser utilizadas como opioides orais para alivio da dor causada pelo câncer, no entanto, deve-se avaliar a condição clínica do paciente, a fim de ofertar o melhor tratamento individual a ele (SCHMIDT-HANSEN M, et al., 2017).

A oximorfona é um opioide de ação curta, por isso, é indicada para o manejo de dor aguda, no entanto, este derivado do ópio é pouco utilizado. Ela está disponível na apresentação de comprimidos de ação curta ou prolongada e pode ser administrada por solução oral ou por via endovenosa (CARDOSO Al, 2013).

O fentanil é um agonista opioide Mu de curta duração, e as suas principais vantagens são o seu início rápido de ação (devido sua alta lipossolubilidade) e a não produção de metabólitos ativos. Comumente, é administrado por via intravenosa, intramuscular ou subcutânea, porém, pode ser usado por via transdérmica e intranasal de acordo com a necessidade do paciente. Ele é de 80 a 100 vezes mais potente que a morfina e o fentanil intranasal, quando comparado à morfina intravenosa, proporciona melhor controle da dor (FONTANELA MA, et al., 2017).

Quando administrado por via transdérmica, o fentanil é um opioide potente de meia vida longa, sendo indicado para pacientes que possuem contraindicação de usar por outra via de administração, ou com intolerância à morfina e com dores constantes. A principal desvantagem desta via é que o início da analgesia demora de 12 a 24 horas após a colocação do adesivo (SILVA LJ, et al., 2020).

A naloxona é um antagonista dos receptores de opioide de ação curta, que atua como antídoto em casos de intoxicação por opioides. Ela é indicada para o tratamento dos casos conhecidos ou suspeitos de sobredosagem dos derivados do ópio, sendo que o principal objetivo desta droga é evitar a depressão respiratória por opioide. Todavia, é necessário observar cuidadosamente o paciente, pois os sintomas de intoxicação por opioide podem reincidir. Ela pode ser administrada por diversas vias, como: parenteral intravenosa, intramuscular e subcutânea, sendo que a administração na mucosa nasal da apresentação da naloxona em spray nasal $(1,8 \mathrm{mg})$ tem sido uma alternativa eficaz, pois evita o efeito de primeira passagem no fígado (LUQUE AG, et al., 2018).

O tratamento dos pacientes com vício em opioides não é fácil, mas podemos dividi-lo em três etapas: estabilização, desintoxicação e manutenção. A primeira etapa consiste na retirada do opioide, optando por outras drogas analgésicas com menos efeitos colaterais. Já a segunda etapa, é a prescrição e administração de drogas antagonistas de opioides, como a naloxona. Por sim, a terceira etapa consiste na prevenção de uma futura sobredosagem de opioide (TORTOSA MJ, et al., 2020).

Sabe-se que o controle da dor em pacientes oncológicos é de extrema importância para a qualidade de vida e para o sucesso do tratamento. Desse modo, é importante que os profissionais de saúde estejam atualizados sobre o melhor manejo das drogas analgésicas, sendo que as principais drogas utilizadas são os opioides, como a morfina ou metadona por via oral; entretanto, nem todos os pacientes obtém analgesia completa ou não toleram os efeitos adversos dos opioides, sendo necessário, então, procedimentos invasivos, como o bloqueio de nervos (JÚNIOR JC, et al., 2016). Além disso, a rotação de opioides é uma alternativa para melhorar a analgesia e diminuir os efeitos colaterais. Ela consiste basicamente em substituir um opioide potente já prescrito por outro opioide potente, alternando a via de administração, por exemplo: trocar a prescrição de meperidina por via intramuscular por morfina por via oral (LÓPES VM, et al., 2019).

A prevalência da dor em pacientes com neoplasias é superior a $75 \%$, o que implica em prejuízos nas atividades diárias do paciente. Porém, para o alívio completo da dor é preciso, além da avaliação da dor e da prescrição de analgésicos, identificar os aspectos psicológicos, sociais, culturais e espirituais da dor, o que torna a intervenção multidisciplinar algo indispensável (MENDES TR, et al., 2014). Nos pacientes não 
oncológicos com dor, como no caso da insuficiência renal e/ou hepática, deve-se manejar as drogas derivadas do ópio levando-se em consideração a sua metabolização e excreção, por exemplo: em paciente com insuficiência renal, os opioides que têm menos risco de gerar complicações são: fentanil, buprenorfina e oximorfina; já em pacientes com insuficiência hepática, drogas como a meperidina e a codeína devem ser evitadas e pode ser necessário aumentar o intervalo ou diminuir a dose de tramadol e morfina (AZEVEDO AS, 2017).

Entende-se que a dor possui vários componentes além do físico, por isso, o plano terapêutico do manejo da dor precisa contemplar uma equipe multidisciplinar. A intervenção de um fisioterapeuta capacitado para atuar nos cuidados paliativos favorece no alivio do sofrimento, além de prevenir deformidades, complicações e estimula o autocuidado (MARQUES CC, 2019).

Igualmente, entende-se que o psicólogo é uma peça chave de grande importância, pois o seu trabalho contribui ativamente para o alívio do componente psicológico da dor; entretanto, isso ainda foge do cenário atual, o que torna necessário uma reformulação e reorganização das equipes de saúde, o que resultaria em um cuidado mais humanitário para os pacientes em cuidado paliativo (BARRETO SM e CASTRO EK, 2015).

Já a equipe de enfermagem é a responsável pelo cuidado individualizado ao paciente, sendo importante para a identificação das causas da dor, administração de drogas analgésicas (especialmente os opioides), cumprimento de horários, e uso de técnicas não farmacológicas para alívio da dor, como a aplicação de calor, mudanças de decúbito e estímulo à deambulação, além disso, é um vínculo ativo com o paciente permitindo acolher imediatamente a demanda do paciente e da família (STUBE M, et al., 2015).

\section{CONSIDERAÇÕES FINAIS}

O manejo de opioides em pacientes de cuidado paliativo é um assunto de extrema importância, pois a dor deles é prejudicial para a qualidade de vida, interferindo nas atividades do cotidiano e comprometendo o plano terapêutico. Dessa forma, o correto manejo dos opioides, bem como a atuação da equipe multidisciplinar, torna-se indispensável para o tratamento dos pacientes paliativos, uma vez que possuem capacidade de minimizar a dor por serem drogas analgésicas potentes. Entretanto, é importante ressaltar que cada paciente deve ser avaliado de forma individual, pois cada um possuirá uma indicação de opioide de acordo com o seu diagnóstico, o que torna o conhecimento médico sobre as formas de metabolização e excreção de cada droga fundamental. Bem como, observa-se a importância de conhecer o mecanismo de ação das drogas por toda a equipe para intervir precocemente nos efeitos adversos e conferir maior segurança na administração.

\section{REFERÊNCIAS}

1. ARAÚJO LG, et al. Cuidados paliativos em pacientes oncológicos: uma abordagem do conhecimento dos enfermeiros. Revista Eletrônica Acervo Saúde, 2020; 12(11): e4663.

2. AZEVEDO AS. Uso de opióides na dor crónica não oncológica: Resistência e Mitos. Dissertação (Mestre em Medicina) - Ciências da Saúde. Universidade da Beira Interior, Covilhã, 2017; 42p.

3. BARBOSA J, et al. Repeated Administration of Clinically Relevant Doses of the Prescription Opioids Tramadol and Tapentadol Causes Lung, Cardiac, and Brain Toxicity in Wistar Rats. Pharmaceuticals (Basel), 2021; $14(2)$ : 97.

4. BARRETO SM, CASTRO EK. Critérios de Médicos Oncologistas para Encaminhamento Psicológico em Cuidados Paliativos. Psicologia: Ciência e Profissão, 2015; 35(1): 69-82.

5. BAO YJ, et al. Hidromorfona for cancer pain. Cochrane Library, 2016; (10): CD011108.

6. BOTAN AG, LAPENA AS. Meperidina: opioide não indicado para analgesia. Rev Dor. São Paulo, 2015; 16(1): 67-70.

7. BRASIL. Ministério da Saúde. 2012.2 Disponível http://bvsms.saude.gov.br/bvs/saudelegis/sas/2012/prt1083_02_10_2012.html. Acessado em 15 de janeiro de 2021.

8. CALÔNEGO MA. Dificuldades sociais, legais e burocráticas para prescrição de opioides. Tese (Pós-graduação em Anestesiologia) - Instituto de Biociências. Universidade Estadual Paulista "Júlio de Mesquita Filho", Botucatu, 2020; 93p.

9. CARDOSO Al. Controlo da dor em pacientes oncológicos. Dissertação (Mestrado Integrado em Medicina) - Instituto de Ciências Biomédicas Abel Salazar. Universidade do Porto, Porto, 2013/2014; 33p.

10. COELHO CB, YANKASKAS JR. New concepts in palliative care in the intensive care unit. Revista Brasileira de Terapia Intensiva, 2017; 29(2): 222-230. 
11. FONTANELA MA, et al. Fentanil intranasal - Revisão de Literatura. Revista de Ciência Veterinária e Saúde Pública. UEM, Umuarama, 2017; 4(2): 111-115.

12. GARCIA LA, et al. Evaluación positiva de medicamentos: septiembre, octubre y noviembre 2017. Sanid. Mi, 2018; 74: 26-31.

13. GONG L, et al. PharmGKB summary: tramadol pathway. Pharmacogenet Genomics, 2014; 24(7): 374-380.

14. GOODMAN A, et al. As bases farmacológicas da terapêutica. 13th ed. Rio de Janeiro: McGraw, 2018; 427-465p.

15. JÚNIOR JC, et al. Analgesia em pacientes com dor crônica oncológica. Sociedade de patologia do Tocantins, 2016; 3(4): 103-111.

16. LEAL RS, ALENCAR GA. Uso indevido de opioides: Da prevenção ao tratamento. Revista de Medicina de família e Saúde mental, 2020; 2: 29-44.

17. LEÓN MX, et al. Recomendaciones basadas en evidencia para el manejo del dolor oncológico (revisión de la literatura). Revista Mexicana de Anestesiología, 2010; 42: 45-55.

18. LÓPES VM, et al. I Manual de Cuidados Paliativos de Extremadura. $1^{\text {st }}$. Extremadura: Junta de Extremadura, 2019; 61-71p.

19. MARQUES CC. Cuidados paliativos: Compreensão de fisioterapeutas que atuam em unidade de terapia intensiva. Dissertação (Curso de especialização em Cuidados Paliativos) - Centro de Ciências da Saúde. Universidade Federal da Paraíba, João Pessoa, 2019; 36p.

20. MENDES TR, et al. Ocorrência da dor nos pacientes oncológicos em cuidado paliativo. Acta Paulista de Enfermagem. 2014; 27(4): 356-361.

21. NAPLES JG, et al. O papel dos analgésicos opióides no tratamento da dor geriátrica. Clinics in geriatric medicine, 2016; 32: 725-735.

22. NETO JO, et al. Revisitando a metadona: farmacocinética, farmacodinâmica e uso clínico. Rev Dor. São Paulo, 2015; 16(1): 60-66.

23. OLIVÊNCIA AS, et al. Tratamento farmacológico da dor crônica não oncológica em idosos: Revisão integrative. Revista Brasileira de Geriatria e Gerontologia, 2018; 21(3): 372-381.

24. ORIOL AL, et al. Antagonistas periféricos de los receptores opioides Mu en el tratamiento del estreñimiento inducido por opioides: revisión. Revista de la Sociedad Española del Dolor, 2018; 27(1): 37-49.

25. OSORIO-GUTIÉRREZ Cl, et al. Comparison among subarachnoid opioid mix for cesarean section - An observational study. Colombian Journal of Anestesiology, 2021; 49(1): e940.

26. PETER YI, PRYZBYLKOWSKI P. Opioid Induced Hyperalgesia. Pain Medicine, 2015; 16: 32-36.

27. PIIRAINEN P, et al. Updated Clinical Pharmacokinetics and Pharmacodynamics of Oxycodone. Clin Pharmacokinet, 2019; 58: 705-725.

28. RANG HP, et al. Farmacologia. 8th ed. Rio de Janeiro: Elsevier, 2016; 510-520p.

29. RIVEROS ME, AYALA SJ. Morfina mitos y realidades: Experiencia en dos centros de Paraguay. An. Fac. Cienc. Méd. (Asunción), 2020; 53(3): 71-80.

30. SANTOS AD, et al. Avaliação farmacoterapêutico de idosos hospitalizados em uso de analgésicos opioides. Revista de Enfermagem do Centro-Oeste Mineiro, 2020; 10: e3665.

31. SANTOS MH. O uso da morfina na gestão da dor crônica: perspectiva dos profissionais de saúde. Dissertação (Mestrado em Cuidados Paliativos) - Instituto Politécnico de Viana Castelo, 2019; 126p.

32. SCHMIDT-HANSEN M, et al. Oxycodone for cancer-related pain. Cochrane Library, 2017; CD003870.

33. SILVA LJ, et al. O uso de opioides no tratamento da dor oncológica em idosos. Brazilian Journal of Pain, São Paulo, 2020; 3(1): 63-72.

34. STUBE M, et al. Percepções de enfermeiros e manejo da dor de pacientes oncológicos. Revista Mineira de Enfermagem. 2015; 19(3): 696-710.

35. TORTOSA MJ, et al. Deshabituación rápida de opioids. Revista de la Sociedad Española del Dolor. 2020; 26(2): 95102.

36. WALTER IT. Avaliação do perfil de prescrição da associação de codeína e paracetamol na atenção básica à saúde em um município catarinense. Dissertação (Graduação em Farmácia) - Centro de Ciências da Saúde. Universidade Federal de Santa Catarina, Florianópolis, 2019; 46p.

37. WIERMANN EG, et al. Consenso Brasileiro sobre Manejo da Dor Relacionada ao Câncer. Revista Brasileira de Oncologia Clínica, 2015; 10: 132-143.

38. WIFFEN PJ, et al. Tramadol with or witout paracetamol (acetaminophen) for cancer pain. Cochrane Library, 2017; 5(5): CD012508. 